\title{
A unified approach to Tianjin trisyllabic tone sandhi: Metrical conditions and tonal complexity
}

\author{
Xiaomei Wang \& Yen-Hwei Lin*
}

\begin{abstract}
Tianjin (Chinese) exhibits complex interactions among its disyllabic tone sandhi rules, leading to both left-to-right and right-to-left rule applications in trisyllabic sequences (cf. Chen 1986, X. Wang 2003). Which directionality to adopt for each particular trisyllabic sequence is arbitrary and cannot be accounted for by any known principles. Based on data from a recent acoustic study, our phonological analysis demonstrates that the seemingly ungoverned directionality is only apparent and that Tianjin tone sandhi rules apply only from left to right when both metrical and tonal complexity conditions are satisfied, thereby providing a unified account.
\end{abstract}

Keywords. Tianjin Chinese; trisyllabic tone sandhi; metrical structure; tonal complexity

1. Introduction. Tianjin Chinese (hereafter Tianjin) exhibits complex interactions among its disyllabic tone sandhi rules, leading not only to inconsistent rule applications (Chen 1986, Milliken et al. 1997) but also to rule underapplication in trisyllabic sequences (X. Wang 2003). No known principle on rule application can account for the conflicting application directionality in Tianjin trisyllabic sequences (Chen 1986). Besides, the underapplication issue cannot even be explained with application directionality. Drawing data from a multispeaker acoustic study (X. Wang \& Lin 2017), we demonstrate that both conflicting directionality and underapplication can be accounted for when metrical conditions on sandhi rule application and differences in tonal complexity are taken into consideration.

Tianjin has four lexical tones: T1(11/21), T2(45/34), T3(213/214) and T4(54), where $1=$ lowest pitch and 5=highest pitch (Chao 1968), and six reported disyllabic tone sandhi rules, as given in (1) and (2). Acoustic data has recently become available (Zhang \& Liu 2011, Wang \& Lin 2017), showing that $\mathrm{T} 1$ has become 41 and the traditionally described $\mathrm{T} 1+\mathrm{T} 1$ rule in (1a) has been changed in current Tianjin speech to T1+T1 $\rightarrow \boldsymbol{T} 2+\mathrm{T} 1$. Since T1 has become 41 , we use Chao number (21) instead of $\mathrm{T} 1$ to indicate the sandhi outputs of T3+T2 and $\mathrm{T} 3+\mathrm{T} 4$ rules.

(1) Four traditional rules (Li \& Liu 1985)
a. $\mathrm{T} 1+\mathrm{T} 1 \rightarrow \boldsymbol{T} 3+\mathrm{T} 1$
cf. T1 + T1 $\rightarrow$ T2 + T1 (Zhang \& Liu 2011)
b. $\mathrm{T} 3+\mathrm{T} 3 \rightarrow \boldsymbol{T} 2+\mathrm{T} 3$
c. $\mathrm{T} 4+\mathrm{T} 1 \rightarrow \boldsymbol{T} 2+\mathrm{T} 1$
d. $\mathrm{T} 4+\mathrm{T} 4 \rightarrow \boldsymbol{T} \boldsymbol{1}+\mathrm{T} 4$

(2) Two newly reported rules (Wee 2004, Zhang \& Liu 2011)
a. $\mathrm{T} 3+\mathrm{T} 2 \rightarrow \boldsymbol{T} \boldsymbol{1}+\mathrm{T} 2$
b. $\mathrm{T} 3+\mathrm{T} 4 \rightarrow \boldsymbol{T} \boldsymbol{1}+\mathrm{T} 4$
cf. T3 + T2 $\rightarrow \mathbf{2 1}+\mathrm{T} 2$ (Wang \& Lin 2017)
$\mathrm{T} 3+\mathrm{T} 4 \rightarrow 21+\mathrm{T} 4$

Zhang \& Liu (2011) and Li \& Chen (2016) indicate that (1d) does not apply in disyllabic sequences. Zhang \& Liu (2011) is a study on Tianjin disyllabic tone sandhi, and therefore it does not include trisyllabic data. Li \& Chen (2016) do not include (1d) in their trisyllabic

\footnotetext{
* We thank Karthik Durvasula, the Michigan State University Phonology-Phonetics Research Group and participants at LSA 2017 for their comments and suggestions. Authors: Xiaomei Wang, Michigan State University (wangxi89@msu.edu) \& Yen-Hwei Lin, Michigan State University (liny@msu.edu).
} 
analysis since (1d) does not apply in disyllabic sequences. Wang \& Lin (2017) also indicate that (1d) does not apply in disyllabic sequences, but it applies in trisyllabic tone sandhi when it is right-aligned ${ }^{1}$.

Tianjin trisyllabic tone sandhi traditionally refers to seven sequences potentially involving two applications of the tone sandhi rules in (1). In previous analyses (e.g. Chen 1986, Milliken et al. 1997), to derive these trisyllabic outputs, the relevant disyllabic rules apply from left to right in some sequences (e.g. T3+T3+T3 $\rightarrow \underline{T 2+\mathrm{T} 3}+\mathrm{T} 3 \rightarrow T 2+T 2+\mathrm{T} 3$ ) and right to left in others (e.g. $\mathrm{T} 1+\mathrm{T} 1+\mathrm{T} 1 \rightarrow \mathrm{T} 1+\underline{T 2+\mathrm{T} 1})$. With two new disyllabic tone sandhi rules in (2), there should be eight more trisyllabic sequences potentially involving two applications of disyllabic tone sandhi. Detailed acoustic data including both sets have recently become available, as summarized in Table 1, which shows only three cases requiring right-to-left rule application.

\begin{tabular}{|l|l|l|l|l|l|}
\hline \multicolumn{2}{|l|}{ a. The seven old combinations } & \multicolumn{3}{l|}{ b. The eight new combinations } \\
\hline Inputs & Outputs & Directionality & Inputs & Outputs & Directionality \\
\hline T1+T4+T4 & T1+T1+T4 & left $\rightarrow$ right & T1+T3+T2 & T1+21+T2 & left $\rightarrow$ right \\
\hline T3+T1+T1 & T3 ${ }^{2}+\boldsymbol{T} 2+\mathrm{T} 1$ & left $\rightarrow$ right & T3+T3+T2 & $\boldsymbol{T 2}+21+\mathrm{T} 2$ & left $\rightarrow$ right \\
\hline T3+T3+T3 & $\boldsymbol{T} 2+\boldsymbol{T} 2+\mathrm{T} 3$ & left $\rightarrow$ right & T4+T3+T2 & T4+21+T2 & left $\rightarrow$ right \\
\hline T1+T1+T1 & T1+T2+T1 & right $\rightarrow$ left & T1+T3+T4 & T1+21+T4 & left $\rightarrow$ right \\
\hline T4+T1+T1 & T4+T2+T1 & right $\rightarrow$ left & T3+T3+T4 & $\boldsymbol{T 2 + 2 1 + T 4}$ & left $\rightarrow$ right \\
\hline T4+T4+T1 & T4+T2+T1 & right $\rightarrow$ left & T3+T4+T1 & $\boldsymbol{T 1 + T 2 + T 1 ~}$ & left $\rightarrow$ right \\
\hline T4+T4+T4 & T4+T1+T4 & neither & T3+T4+T4 & $\boldsymbol{T 1}+\boldsymbol{T 1}+\mathrm{T} 4$ & left $\rightarrow$ right \\
\hline & & & T4+T3+T4 & T4+21+T4 & left $\rightarrow$ right \\
\hline
\end{tabular}

Table 1 Results of the acoustic study for fifteen trisyllabic sequences (Wang \& Lin 2017)

2. Conflicting directionality and underapplication. The two issues to be dealt with in this paper are conflicting directionality and underapplication in Tianjin trisyllabic tone sandhi. The application directionality to derive attested output is not consistent. In some trisyllabic sequences, the disyllabic sandhi rules have to apply from left to right (3), but in some other sequences, the rules have to apply from right to left, as shown in (4), where UR = underlying representation and $\mathrm{SR}=$ surface representation.

(3) Some patterns are derived from left to right

$\begin{array}{ll}\mathrm{T} 1+\mathrm{T} 4+\mathrm{T} 4 \rightarrow \mathrm{T} 1+\boldsymbol{T} 1+\mathrm{T} 4 \\ \text { a. } \begin{array}{l}\text { Left to right } \rightarrow \\ \text { AR }\end{array} & \text { Ttested output } \\ \text { No rule } & \underline{\mathrm{T} 1+\mathrm{T} 4+\mathrm{T} 4}+\mathrm{T} 4 \\ \mathrm{~T} 4+\mathrm{T} 4 \text { rule } & \mathrm{T} 1+\boldsymbol{T} 1+\mathrm{T} 4 \\ \text { SR } & \mathrm{T} 1+\boldsymbol{T} 1+\mathrm{T} 4\end{array}$

\footnotetext{
There are on-going changes in Tianjin tone sandhi (X. Wang 2015). The application rates of the T4+T4 rule are very low among young Tianjin speakers, but are comparatively high among old Tianjin speakers, which indicates that the application of the T4+T4 rule is decreasing (X. Wang 2015). Although the T4+T4 sandhi pattern almost disappears among young speakers in their wordlist reading style, it still exists in the same speakers' less careful speech in the interview style (X. Wang 2015). This can explain why in our acoustic data, the T4+T4 rule does not apply in disyllabic sequences, but still applies in trisyllabic ones when the sequence is right-aligned.

2 The output of T3 is reduced, which preserves a small rise from the underlying T3. Wang \& Lin (2017) indicate that it is 212 .
} 

b. Right to left $\rightarrow$ Unattested output
UR $\quad \mathrm{T} 1+\mathrm{T} 4+\mathrm{T} 4$
$\mathrm{T} 4+\mathrm{T} 4$ rule $\quad \mathrm{T} 1+\boldsymbol{T} 1+\mathrm{T} 4$
$\mathrm{T} 1+\mathrm{T} 1$ rule $\quad \boldsymbol{T} 2+\overline{\boldsymbol{T} 1+\mathrm{T} 4}$
$\mathrm{SR} \quad * \boldsymbol{T} \mathbf{2}+\boldsymbol{T} 1+\mathrm{T} 4$

(4) Some patterns are derived from right to left

$\mathrm{T} 4+\mathrm{T} 4+\mathrm{T} 1 \rightarrow \mathrm{T} 4+\boldsymbol{T} 2+\mathrm{T} 1$

a. Right to left $\rightarrow$ Attested output

$\begin{array}{ll}\mathrm{UR} & \mathrm{T} 4+\mathrm{T} 4+\mathrm{T} 1 \\ \mathrm{~T} 4+\mathrm{T} 1 \text { rule } & \mathrm{T} 4+\underline{\boldsymbol{T} 2}+\mathrm{T} 1 \\ \mathrm{~T} 4+\mathrm{T} 4 \text { rule } & ---------- \\ \mathrm{SR} & \mathrm{T} 4+\boldsymbol{T} 2+\mathrm{T} 1\end{array}$

b. Left to right $\rightarrow$ Unattested output

UR T4+T4+T1

T4+T4 rule $\quad \boldsymbol{T} 1+\mathrm{T} 4+\mathrm{T} 1$

$\mathrm{T} 4+\mathrm{T} 1$ rule $\quad \overline{\boldsymbol{T} \boldsymbol{1}+\underline{\boldsymbol{T} 2}+\mathrm{T} 1}$

$\mathrm{SR} \quad * \boldsymbol{T} \mathbf{1}+\boldsymbol{T} 2+\mathrm{T} 1$

The second issue to resolve is rule underapplication. Some disyllabic tone sandhi rules do not apply in some trisyllabic sequences even when their sandhi conditions are satisfied. For example, the attested output of T4+T4+T4 is T4+T1+T4. From neither direction can this attested output be derived. If the directionality of rule application goes from left to right, the output would be $* \mathrm{~T} 1+\mathrm{T} 1+\mathrm{T} 4$. If the directionality goes from right to left, the output would be $* \mathrm{~T} 2+\mathrm{T} 1+\mathrm{T} 4$. To derive the attested output, either from left to right or right to left, one of the relevant disyllabic rules must not apply even when its sandhi condition is satisfied, as the derivations in (5) illustrate.

(5) Underapplication

$\mathrm{T} 4+\mathrm{T} 4+\mathrm{T} 4 \rightarrow \mathrm{T} 4+\mathrm{T} 1+\mathrm{T} 4$

a. Left to right rule application

$\begin{array}{lll}\text { UR } & \mathrm{T} 4+\mathrm{T} 4+\mathrm{T} 4 & \\ \text { T4+T4 rule } & \frac{\mathrm{T} 4+\mathrm{T} 4+\mathrm{T} 4}{\mathrm{~T} 4+\boldsymbol{T} 1}+\mathrm{T} 4 & \text { no application } \\ \text { T4+T4 rule } & \mathrm{T} 4 \text { plication } \\ \text { SR } & \mathrm{T} 4+\boldsymbol{T} 1+\mathrm{T} 4 & \end{array}$

b. Right to left rule application

UR T4+T4+T4

$\mathrm{T} 4+\mathrm{T} 4$ rule $\quad \mathrm{T} 4+\underline{T} 1+\mathrm{T} 4 \quad$ application

$\mathrm{T} 4+\mathrm{T} 1$ rule $\quad \mathrm{T} 4+\mathrm{T} 1+\mathrm{T} 4 \quad$ no application

SR

$\mathrm{T} 4+\boldsymbol{T} 1+\mathrm{T} 4$

We propose an analysis of Tianjian trisyllabic tone sandhi in which metrical structure and disyllabic sandhi rules interact, and demonstrate that Tianjin tone sandhi rules apply consistently from left to right when both metrical and tonal complexity conditions are satisfied, thereby removing the need for conflicting directionality and solving the underapplication issue.

3. Phonological analysis. Metrical structure and stress have strong influences on tone sandhi (Wright 1983, Duanmu 1995, Chan 1985, Wang \& Lin 2016, among others). Wright (1983) argues that in Fuzhou Chinese and Shanghai Chinese, the weakly stressed syllables are shown to have shortened duration and loss of original tonal contour. Duanmu (1995) attributes the tonal stability in Mandarin and the lack of tonal stability in Shanghai to the 
difference in stress. In Mandarin, each regular syllable is heavy and forms a bimoraic foot, and as a result, the syllable keeps its underlying tone. In Shanghai, since only initial syllables have stress, all non-initial syllables are unstressed and cannot keep their underlying tones. Wang \& Lin (2016) indicates that disyllabic tonal domain in Ei language is iambic and tonal reduction occurs in the non-head syllable. Different from the New Shanghai in Duanmu (1995), no tone spread occurs in Ei language and there is no phonological influence from the head-syllable tone on the non-head syllable. Instead, the reduced tone surfaces as a lowregister tone with variable phonetic pitch values that seem to reflect some residual phonetic properties of the original tone.

These studies indicate that stressed syllables tend to keep their original tones while unstressed syllables have a tendency to lose their original tones, which is in agreement with our analysis here. In disyllabic sequences, unless the second syllable is a neutral tone, the first syllable loses its underlying tone because Tianjin disyllables form right-headed feet ( $\mathrm{J}$. Wang 2002, Wee 2015). In trisyllables, unless the last syllable is a neutral tone, the first or second syllable or both, depending on tonal complexity, lose their underlying tones while the prosodic head (the third syllable) keeps its original tone. For detailed discussion on Tianjin metrical structures, see subsection 3.1.

Prosodic prominence and tonal complexity are correlated (Hyman 2007, Yip 2002:2730), which is also important to our analysis. Contour tones like T3 (214) in Tianjin need longer duration, and as a result, T3 cannot keep its underlying tone unless it is on the prosodic head. Contour tones occur more freely in stressed syllables than unstressed syllables, with no language display the opposite pattern (Zhang 2002).

In this section, we show how metrical structure and tonal complexity account for the application inconsistency in $\mathrm{T} 4+\mathrm{T} 1, \mathrm{~T} 4+\mathrm{T} 4$, and $\mathrm{T} 1+\mathrm{T} 1$, and the application consistency in $\mathrm{T} 3+\mathrm{T} 2, \mathrm{~T} 3+\mathrm{T} 3$ and $\mathrm{T} 3+\mathrm{T} 4$ in Tianjin trisyllabic sequences. The conflicting directionality and underapplication issues discussed in $\S 2$ are originated from the inconsistent application of $\mathrm{T} 4+\mathrm{T} 1, \mathrm{~T} 4+\mathrm{T} 4$ and $\mathrm{T} 1+\mathrm{T} 1$, and the consistent application of $\mathrm{T} 3+\mathrm{T} 2, \mathrm{~T} 3+\mathrm{T} 3$ and $\mathrm{T} 3+\mathrm{T} 4$. The root of irregular directionality is that some disyllabic tone sandhi rules do not apply even when their sandhi conditions are satisfied. For example, $\mathrm{T} 1+\mathrm{T} 1+\mathrm{T} 1$ is one of the three rightto-left application patterns. To derive the attested output, the left-aligned T1+T1 rule cannot apply even when its sandhi condition is satisfied, as shown in (6a). Right-to-left application directionality is adopted in traditional rule-based analysis to account for the non-application on the left-aligned $\mathrm{T} 1+\mathrm{T} 1$ sequence because the right-to-left directionality ruins the sandhi condition of the left-aligned $\mathrm{T} 1+\mathrm{T} 1$, as shown in (6b). But even with conflicting application directionality, underapplication issue in $\mathrm{T} 4+\mathrm{T} 4+\mathrm{T} 4$ remains unresolved.

(6) $\mathrm{T} 1+\mathrm{T} 1+\mathrm{T} 1 \rightarrow \mathrm{T} 1+\mathrm{T} 2+\mathrm{T} 1$

a. Left to right rule application

UR $\quad \mathrm{T} 1+\mathrm{T} 1+\mathrm{T} 1$

$\mathrm{T} 1+\mathrm{T} 1$ rule $\quad \underline{T 2}+\mathrm{T} 1+\mathrm{T} 1$

$\mathrm{T} 1+\mathrm{T} 1$ rule $\quad \underline{\mathrm{T} 2+\boldsymbol{T} 2}+\mathrm{T} 1$

$\mathrm{SR} \quad * \boldsymbol{T} \boldsymbol{2}+\boldsymbol{T} \boldsymbol{T}+\mathrm{T} 1$

b. Right to left rule application

UR T1+T1+T1

$\mathrm{T} 1+\mathrm{T} 1$ rule $\mathrm{T} 1+\underline{T} 2+\mathrm{T} 1$

$\mathrm{T} 1+\mathrm{T} 1$ rule

SR

$\mathrm{T} 1+\boldsymbol{T} 2+\mathrm{T} 1$ 
By taking metrical structures into consideration, we can account for the inconsistent application of the $\mathrm{T} 4+\mathrm{T} 1, \mathrm{~T} 4+\mathrm{T} 4$ and $\mathrm{T} 1+\mathrm{T} 1$ rules, and therefore remove the need of conflicting directionality. Our proposed analysis can be summarized as follows: (i) the directionality of rule application is consistently from left to right; (ii) a T1(41) or T4(53) occupying a foot head does not undergone tone sandhi; (iii) the most complex tone T3(214) does not undergo tone sandhi only when it occupies the prosodic head or primary stress. Since metrical structure is a key factor in resolving the two issues in Tianjin trisyllabic tone sandhi, Tianjin trisyllabic metrical structures will be introduced first in the next subsection, followed by additional subsections on different aspects of and evidence for our proposed analysis.

3.1. TIANJIN METRICAL STRUCTURES. Given the observation that the second tone in all Tianjin disyllabic tone sandhi keeps their underlying tones, Tianjin disyllabic sequences are rightheaded when both syllables are full-toned (J. Wang 2002, Wee 2015). The position where neutral tone appears in disyllables in Tianjin seems to be contradictory to the rightheadedness since neutral toned syllables never appear initially in disyllabic sequences and neutral tones are much weaker than any full tones in Tianjin. Z. Wang (1999) points out that neutral-toned syllables involve morphological changes. The neutral-toned syllable may be a suffix or result from tonal reduction on a fully-toned syllable leading to syntactic or semantic changes. Similar ideas were expressed in Wee (2015).

Tianjin trisyllabic metrical structure has been claimed to be [secondary stress, weak, primary stress] when the last syllable is not a neutral tone (Huang, Yan and Lu 2005), as given in (7), in which 1 stands for the primary stress, 2 stands for the secondary stress and 3 stands for the weakest syllable. Morpho-syntactic structures are irrelevant in this metrical pattern.

(7) Tianjin Trisyllabic Metrical Pattern

$\begin{array}{ccl}\sigma & \sigma & \sigma \\ (\mathrm{x}) & (\mathrm{x} & \mathrm{x}) \\ (\mathrm{x} & & \mathrm{x}) \\ & & \mathrm{x} \\ \text { tu } & \text { shu } & \text { guan (library) } \\ 2 & 3 & 1\end{array}$

Tianjin speakers sometimes reduce or delete syllables in multi-syllabic utterances in casual, friendly conversations, which is called syllable swallowing. Huang, Yan \& Lu (2005) investigate 570 tri- and quadro- syllabic utterances familiar to their informant and found systemic patterns in syllable swallowing. Their conclusion is that stability in syllabic positions is ordered: final syllables are most stable; initial syllables less so; medial syllables most volatile. They attribute the stability order in trisyllables to the stress pattern. The weak syllable tends to lose its underlying form, while the strong syllable tends to keep its underlying form. In Tianjin trisyllabic sequences, the last syllable is the strongest, and therefore it is the most stable. The medial syllable is the weakest, and therefore it tends to lose its underlying form.

The observation from Huang, Yan and Lu (2005) is supported by our data. We investigated 321 trisyllabic utterances taken from sociolinguistic interviews recorded in the summers of 2014-2016. All the swallowed syllables are in the second syllable, while the first and the last syllables remain complete.

Studies on Beijing Chinese stress patterns also indicate that the second syllable is the weakest in trisyllables. The stress pattern of Tianjin is the same as the stress pattern of Beijing (Huang, Yan \& Lu 2005). Both Tianjin and Beijing Chinese belong to Northern Mandarin. Existing studies have different views on whether the first syllable or the last 
syllable is the heaviest or prosodically most prominent in Beijing Chinese trisyllabic sequences, but all studies agree that the second syllable is the weakest. The Beijing stress pattern is [secondary stress, weak, primary stress] in Chao (1968) and Yan \& Lin (1988), but [primary stress, weak, secondary stress] in J. Wang \& L. Wang (1993).

In addition to phonological and typological evidence cited above, we also have phonetic evidence to support the [secondary stress, weak, primary stress] metrical structure in Tianjin trisyllabic sequences. We measured the duration of each syllable in trisyllabic sequences and found that the last syllable is the longest while the second is the shortest when other conditions are held the same.

Previous studies on Tianjin neutral tone indicate that duration is the most important acoustic correlate of stress in Tianjin, and therefore we measured the duration of trisyllables to provide further evidence for the stress pattern. In Tianjin, the duration of a neutral syllable is only about half of the duration of a full syllable (J. Wang 2002, Lu \& J. Wang 2012). Any neutral tone is much shorter and weaker than any full tone. When the duration of a full syllable is reduced to about half of its original duration, that syllable is perceived as a neutral tone (Lu \& J. Wang 2012).

Because the durations of the four lexical tones are inherently different, ideally we should measure the trisyllabic sequences having the same output tones in all the three syllables. Because of rich disyllabic tone sandhi changes in Tianjin, we could not find any trisyllabic pattern having the same output tones in all the three syllables. So we have to compare the first two syllables and the last two syllables respectively. We selected the $\mathrm{T} 1+\mathrm{T} 1+\mathrm{T} 1, \mathrm{~T} 4+\mathrm{T} 4+\mathrm{T} 4, \mathrm{~T} 3+\mathrm{T} 3+\mathrm{T} 3$ and $\mathrm{T} 1+\mathrm{T} 4+\mathrm{T} 4$ patterns. The output of $\mathrm{T} 1+\mathrm{T} 1+\mathrm{T} 1$ is $\mathrm{T} 1+\mathrm{T} 2+\mathrm{T} 1$, and the output of $\mathrm{T} 4+\mathrm{T} 4+\mathrm{T} 4$ is $\mathrm{T} 4+\mathrm{T} 1+\mathrm{T} 4$, where the output tones of the first syllable and the third syllable are the same in each pattern. The output of $\mathrm{T} 3+\mathrm{T} 3+\mathrm{T} 3$ is $\mathrm{T} 2+\mathrm{T} 2+\mathrm{T} 3$ and the output of $\mathrm{T} 1+\mathrm{T} 4+\mathrm{T} 4$ is $\mathrm{T} 1+\mathrm{T} 1+\mathrm{T} 4$, where the output tones of the first syllable and second syllable are the same in each pattern. In order to minimize boundary effects, the trisyllabic sequences are set in a carrier sentence "I read XXX three times".

The results indicate that the first syllable is longer than the second syllable in the $\mathrm{T} 3+\mathrm{T} 3+\mathrm{T} 3 \rightarrow \mathrm{T} 2+\mathrm{T} 2+\mathrm{T} 3$ and $\mathrm{T} 1+\mathrm{T} 4+\mathrm{T} 4 \rightarrow \mathrm{T} 1+\mathrm{T} 1+\mathrm{T} 4$ patterns and the third syllable is longer than the first syllable in the $\mathrm{T} 1+\mathrm{T} 1+\mathrm{T} 1 \rightarrow \mathrm{T} 1+\mathrm{T} 2+\mathrm{T} 1$ and $\mathrm{T} 4+\mathrm{T} 4+\mathrm{T} 4 \rightarrow \mathrm{T} 4+\mathrm{T} 1+\mathrm{T} 4$ patterns. Paired $t$ test indicates that the difference between the duration of the first syllable and the second syllable is significant $(\mathrm{p}<0.05)$, and the different between the first syllable and the third syllable is significant too $(p<0.05)$. The difference between the first syllable and the second syllable is very consistent, but the difference between the first syllable and the third syllable shows interspeaker and intraspeaker variations.

Our phonetic analyses support the view that the second syllable is the weakest and the third syllable is the strongest in trisyllables. The [secondary stress, weak, primary stress] metrical structure is further supported by the tone sandhi changes in trisyllables ending with a neutral tone. The metrical structure of a trisyllable ending with a neutral tone is different from that of the trisyllable ending with a full tone, because neutral tone is unstressed or extrametrical. The metrical structure of a trisyllabic sequence ending in a neutral tone is shown in (8).

(8) Tianjin trisyllabic metrical structure with a final neutral tone

$\begin{array}{lll}\sigma & \sigma & \sigma_{\mathrm{N}} \\ (\mathrm{x} & \mathrm{x}) & <\mathrm{x}> \\ & \mathrm{x} & \end{array}$


The sandhi outputs of trisyllables ending with a neutral tone are different from the outputs of trisyllables ending with a full tone when other conditions are the same (Wang \& Lin 2017). For example, in $\mathrm{T} 4+\mathrm{T} 1+\mathrm{X}(\mathrm{X}=$ any full tone), $\mathrm{T} 4$ does not change even when its sandhi condition is satisfied. But in $\mathrm{T} 4+\mathrm{T} 1+\mathrm{N}(\mathrm{N}=$ neutral tone), $\mathrm{T} 4$ undergoes sandhi change. The inconsistency of the sandhi change of T4+T1 can be accounted for when metrical conditions are incorporated into the analysis, and the inconsistency of the sandhi change itself is also evidence to support the metrical structure analyses. A detailed analysis of trisyllables ending with a neutral tone is given in subsection 3.4

3.2 CONFLICTING DiRECTIONALITY. The seemingly irregular directionality disappears if metrical condition is taken into consideration. Of all the fifteen trisyllabic sequences we have analyzed, only three are derived from right to left $(\mathrm{T} 1+\mathrm{T} 1+\mathrm{T} 1, \mathrm{~T} 4+\mathrm{T} 1+\mathrm{T} 1$ and $\mathrm{T} 4+\mathrm{T} 4+\mathrm{T} 1)$. One crucial generalization is that for these three sequences to derive the attested outputs, the tone of the first syllable cannot be changed even when its sandhi condition is satisfied. Traditional analyses with right-to-left application can block the sandhi application of the leftaligned $\mathrm{T} 1+\mathrm{T} 1 \mathrm{~T} 4+\mathrm{T} 1$, and $\mathrm{T} 4+\mathrm{T} 4$, but results in directionality in conflict with the majority of other trisyllabic sequences. Our metrically-conditioned analysis maintains that the application directionality is consistently from left to right, and a T1 or T4 occupying a foot head does not undergo tone sandhi. The derivations of these three sequences are given in (911), where MS = metrical structure.

In (9), when $\mathrm{T} 1+\mathrm{T} 1$ is left-aligned, the first $\mathrm{T} 1$ is on a stressed syllable and therefore keeps its original tone. When $\mathrm{T} 1+\mathrm{T} 1$ is right-aligned, the first $\mathrm{T} 1$ is on the weak syllable and therefore loses its original tone. For the same reason, T4+T1 and T4+T4 do not undergo sandhi when they are left-aligned, but they do when they are right-aligned, as show in (10) and (11). Our metrically-conditioned analysis can account for the inconsistent application to $\mathrm{T} 1+\mathrm{T} 1, \mathrm{~T} 4+\mathrm{T} 1$ and $\mathrm{T} 4+\mathrm{T} 4$ in trisyllables without resorting to different rule application directionalities. $\mathrm{T} 1+\mathrm{T} 1, \mathrm{~T} 4+\mathrm{T} 1$ and $\mathrm{T} 4+\mathrm{T} 4$ undergo sandhi inconsistently in different positions in trisyllables because their metrical conditions are different when they are rightaligned versus when they are left-aligned. $\mathrm{T} 1+\mathrm{T} 1, \mathrm{~T} 4+\mathrm{T} 1$ and $\mathrm{T} 4+\mathrm{T} 4$ fail to undergo sandhi when they are left-aligned because their metrical conditions are not satisfied.

(9) $\mathrm{T} 1+\mathrm{T} 1+\mathrm{T} 1 \rightarrow \mathrm{T} 1+\mathrm{T} 2+\mathrm{T} 1$ with left-to-right rule application

\begin{tabular}{|c|c|c|}
\hline \multirow{3}{*}{$\begin{array}{l}\text { UR } \\
\text { MS }\end{array}$} & $\mathrm{T} 1+\mathrm{T} 1+\mathrm{T} 1$ & \\
\hline & $(\mathrm{x})(\mathrm{x} \quad \mathrm{x})$ & \\
\hline & x) & \\
\hline & $\begin{array}{c}\mathrm{X} \\
-101+10\end{array}$ & \\
\hline $\mathrm{T} 1+\mathrm{T} 1$ rule & $\underline{\mathrm{T} 1+\mathrm{T} 1+\mathrm{T} 1}$ & no application; first $\mathrm{T} 1$ on foot head \\
\hline $\mathrm{T} 1+\mathrm{T} 1$ rule & $\mathrm{T} 1+\underline{\boldsymbol{T} 2+\mathrm{T} 1}$ & application \\
\hline & $\mathrm{T} 1+\boldsymbol{T} \boldsymbol{2}+\mathrm{T} 1$ & \\
\hline
\end{tabular}

(10) $\mathrm{T} 4+\mathrm{T} 1+\mathrm{T} 1 \rightarrow \mathrm{T} 4+\mathrm{T} 2+\mathrm{T} 1$ with left-to-right rule application

\begin{tabular}{|c|c|c|}
\hline \multirow{4}{*}{$\begin{array}{l}\text { UR } \\
\text { MS }\end{array}$} & $\mathrm{T} 4+\mathrm{T} 1+\mathrm{T} 1$ & \\
\hline & $(x)(x \quad x)$ & \\
\hline & $\left(\begin{array}{ll}\mathrm{x} & \mathrm{x}\end{array}\right)$ & \\
\hline & $\mathrm{x}$ & \\
\hline $\mathrm{T} 4+\mathrm{T} 1$ rule & $\mathrm{T} 4+\mathrm{T} 1+\mathrm{T} 1$ & no application; $\mathrm{T} 4$ on foot head \\
\hline $\mathrm{T} 1+\mathrm{T} 1$ rule & $\overline{\mathrm{T} 4+\boldsymbol{T} \boldsymbol{2}+\mathrm{T} 1}$ & application \\
\hline & $\mathrm{T} 4+\overline{\boldsymbol{T} 2+\mathrm{T} 1}$ & \\
\hline
\end{tabular}


(11) $\mathrm{T} 4+\mathrm{T} 4+\mathrm{T} 1 \rightarrow \mathrm{T} 4+\mathrm{T} 2+\mathrm{T} 1$ with left-to-right rule application

\begin{tabular}{|c|c|c|}
\hline UR & $\mathrm{T} 4+\mathrm{T} 4+\mathrm{T} 1$ & \\
\hline MS & $(\mathrm{x})(\mathrm{x} \quad \mathrm{x})$ & \\
\hline & $\left(\begin{array}{ll}(x & x\end{array}\right)$ & \\
\hline $4+\mathrm{T} 4$ rule & $\underline{\mathrm{T} 4+\mathrm{T} 4}+\mathrm{T} 1$ & no application; first $\mathrm{T} 4$ on foot head \\
\hline +T1 rule & $\mathrm{T} 4+\underline{\boldsymbol{T} 2}+\mathrm{T} 1$ & application \\
\hline & $\mathrm{T} 4+\boldsymbol{T} \boldsymbol{2}+\mathrm{T} 1$ & \\
\hline
\end{tabular}

In sum, in addition to tonal conditions, metrical conditions have to be satisfied before a disyllabic sandhi rule can apply. The $\mathrm{T} 1+\mathrm{T} 1, \mathrm{~T} 4+\mathrm{T} 1$ and $\mathrm{T} 4+\mathrm{T} 4$ rules do not apply when the relevant sequences are left-aligned since their metrical conditions are not satisfied. When the sequences are right-aligned, their metrical conditions are satisfied, and therefore the relevant rules apply. The application inconsistency of $\mathrm{T} 1+\mathrm{T} 1, \mathrm{~T} 4+\mathrm{T} 1$ and $\mathrm{T} 4+\mathrm{T} 4$ in trisyllables can be explained with metrical conditions included in the analysis.

3.3. UNDERAPPLICATION ISSUE. To derive the attested output of $\mathrm{T} 4+\mathrm{T} 4+\mathrm{T} 4 \rightarrow \mathrm{T} 4+\mathrm{T} 1+\mathrm{T} 4$, either from left to right or from right to left, one of the relevant disyllabic sandhi rules must not apply, as shown in (12), repeated from (5) in $\$ 2$.

(12) Underapplication in $\mathrm{T} 4+\mathrm{T} 4+\mathrm{T} 4$

$\mathrm{T} 4+\mathrm{T} 4+\mathrm{T} 4 \rightarrow \mathrm{T} 4+\mathrm{T} 1+\mathrm{T} 4$

a. Left to right rule application

UR T4+T4+T4

$\mathrm{T} 4+\mathrm{T} 4$ rule $\quad \underline{\mathrm{T} 4+\mathrm{T} 4}+\mathrm{T} 4 \quad$ no application

$\mathrm{T} 4+\mathrm{T} 4$ rule $\mathrm{T} 4+\boldsymbol{T} 1+\mathrm{T} 4 \quad$ application

SR $\quad \mathrm{T} 4+\boldsymbol{T} 1+\mathrm{T} 4$

b. Right to left rule application

UR T4+T4+T4

$\mathrm{T} 4+\mathrm{T} 4$ rule $\mathrm{T} 4+\underline{\boldsymbol{T} 1+\mathrm{T} 4} \quad$ application

$\mathrm{T} 4+\mathrm{T} 1$ rule $\mathrm{T} 4+\mathrm{T} 1+\mathrm{T} 4$ no application

SR $\quad \mathrm{T} 4+\boldsymbol{T} 1+\mathrm{T} 4$

In order to derive the attested output, the $\mathrm{T} 4+\mathrm{T} 1$ or $\mathrm{T} 4+\mathrm{T} 4$ rule cannot apply even though their tone sandhi conditions are satisfied. This underapplication issue can be resolved because $\mathrm{T} 4$ or $\mathrm{T} 1$ does not undergo tone sandhi when they are on a foot head (i.e. has a secondary stress). Our metrically-conditioned analysis is illustrated in (13), which follows the same analysis as in the previous subsection.

(13) $\mathrm{T} 4+\mathrm{T} 4+\mathrm{T} 4 \rightarrow \mathrm{T} 4+\mathrm{T} 1+\mathrm{T} 4$ with left-to-right rule application

\begin{tabular}{|c|c|c|}
\hline \multirow{3}{*}{$\begin{array}{l}\text { UR } \\
\text { MS }\end{array}$} & $\mathrm{T} 4+\mathrm{T} 4+\mathrm{T} 4$ & \\
\hline & $(x)\left(\begin{array}{ll}x & x\end{array}\right)$ & \\
\hline & $\left(\begin{array}{ll}\mathrm{x} & \mathrm{x}\end{array}\right)$ & \\
\hline & $\mathrm{x}$ & \\
\hline $4+\mathrm{T} 4$ rule & $\underline{\mathrm{T} 4}+\mathrm{T} 4+\mathrm{T} 4$ & no application; first $\mathrm{T} 4$ on foot head \\
\hline $4+\mathrm{T} 4$ rule & $\overline{\mathrm{T}} 4+\underline{T} \boldsymbol{1}+\mathrm{T} 4$ & application \\
\hline & $\mathrm{T} 4+\overline{\boldsymbol{T} 1+\mathrm{T} 4}$ & \\
\hline
\end{tabular}

When T4+T4 is left-aligned, the first T4 is on a stressed syllable/foot head, and hence cannot undergo tone sandhi. On the other hand, for the right-aligned T4+T4, the middle T4 in the trisyllabic sequence is on the weak syllable, and thus undergoes tone sandhi. 
3.4. GENERALIZATIONS AND FURTHER EVIDENCE FOR THE METRICAL EFFECTS. X. Wang (2003) found that the T4+T1 rule does not apply when the T4+T1 sequence is left-aligned, but duly applies when the sequence is right-aligned. The present study expands this same generalization to both $\mathrm{T} 1+\mathrm{T} 1$ and $\mathrm{T} 4+\mathrm{T} 4$ rules; that is, the three $\mathrm{T} 4+\mathrm{T} 1, \mathrm{~T} 1+\mathrm{T} 1$ and $\mathrm{T} 4+\mathrm{T} 4$ rules do not apply when the relevant sequences are left-aligned, but regularly apply when the sequences are right-aligned. Li \& Chen (2016) also showed that T1+T1 and T4+T1 sandhi apply only when they are right-aligned, but they did not explain why. The left-aligned patterns are exactly those requiring right-to-left rule application and/or blocking rule application in the traditional analysis. The derivations in (14) show how metrical structure can account for this discrepancy, using T4+T1 as the example.
a. Left-aligned patterns
UR $\mathrm{T} 4+\mathrm{T} 1+\mathrm{X}$ $\mathrm{T} 4+\mathrm{T} 1+\mathrm{X}$ no rule application
SR $\quad \mathrm{T} 4+\mathrm{T} 1+\mathrm{X}$
$(\mathrm{x})(\mathrm{x} \quad \mathrm{x})$
$\left(\begin{array}{ll}\mathrm{x} & \mathrm{x}\end{array}\right)$
$\mathrm{x}$

b. Right-aligned patterns

$\begin{array}{ll}\text { UR } & \mathrm{X}+\mathrm{T} 4+\mathrm{T} 1 \\ & \mathrm{X}+\boldsymbol{T} 2+\mathrm{T} 1 \\ & \text { rule application } \\ \mathrm{SR} & \mathrm{X}+\boldsymbol{T} 2+\mathrm{T} 1\end{array}$

When T4+T1 is left-aligned in a trisyllabic sequence, T4 is on a stressed syllable/foot head, and therefore T4 does not undergo tone sandhi. When T4+T1 is right-aligned, T4 is on a weak syllable, and therefore undergoes tone sandhi.

Further evidence for the metrical effects on Tianjin trisyllabic tone sandhi comes from trisyllabic sequences with a final neutral tone. In a $\mathrm{T} 4+\mathrm{T} 1+\mathrm{N}$ sequence where the metrical structure becomes [weak-strong-weak], left-aligned T4+T1 undergoes sandhi since T4 is in a weak position. The metrical structure of $\mathrm{T} 4+\mathrm{T} 1+\mathrm{N}$ is different from that of $\mathrm{T} 4+\mathrm{T} 1+\mathrm{X}$ as the neutral tone is unstressed or extrametrical. The metrical structure of trisyllabic sequences ending with a neutral tone was given in (8) earlier, and is restated here as (15) for ease of reference.

(15) Tianjin trisyllabic metrical structure with a final neutral tone

$\begin{array}{llr}\sigma & \sigma & \sigma_{N} \\ (\mathrm{x} & \mathrm{x}) & <\mathrm{x}> \\ & \mathrm{x}\end{array}$

We take $\mathrm{T} 4+\mathrm{T} 1+\mathrm{X}$ and $\mathrm{T} 4+\mathrm{T} 1+\mathrm{N}$ as examples in (16) to show how differences in metrical structures affect the application of Tianjin tone sandhi. In both $\mathrm{T} 4+\mathrm{T} 1+\mathrm{X}$ and $\mathrm{T} 4+\mathrm{T} 1+\mathrm{N}$ patterns, T4+T1 is left-aligned. What is interesting is that $\mathrm{T} 4$ undergoes tone sandhi in $\mathrm{T} 4+\mathrm{T} 1+\mathrm{N}$ but remains unchanged in $\mathrm{T} 4+\mathrm{T} 1+\mathrm{X}$. Since the metrical structure of $\mathrm{T} 4+\mathrm{T} 1+\mathrm{N}$ is different from that of $\mathrm{T} 4+\mathrm{T} 1+\mathrm{X}, \mathrm{T} 4$ is on a weak syllable in $\mathrm{T} 4+\mathrm{T} 1+\mathrm{N}$, and undergoes tone sandhi. The application versus non-application follows our analysis in which T1 and T4 undergo tone sandhi only when they occupy a weak syllable. 


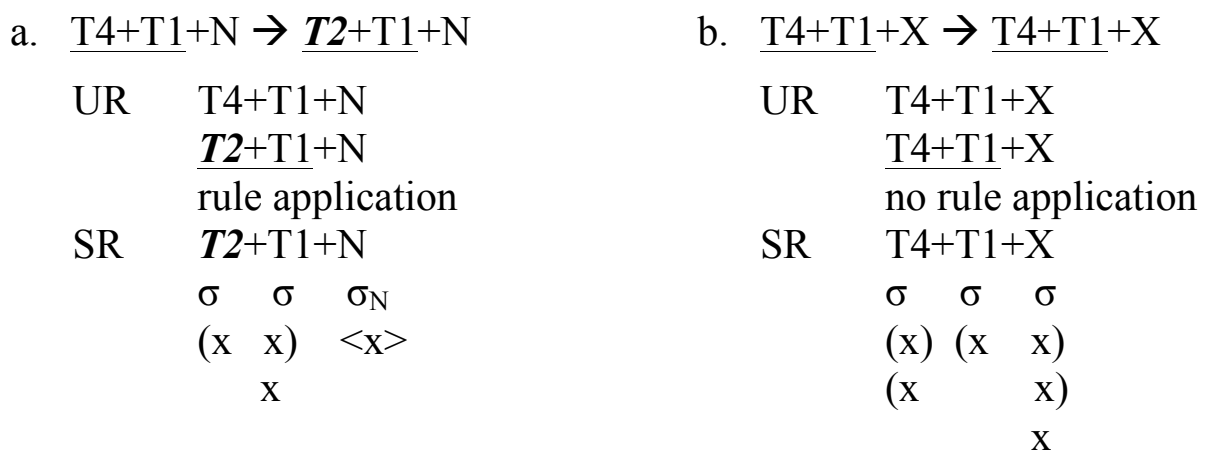

3.5. TONAL COMPLEXITY. Our metrically-conditioned analysis raises a question regarding the consistent application of $\mathrm{T} 3+\mathrm{T} 3, \mathrm{~T} 3+\mathrm{T} 2$ and $\mathrm{T} 3+\mathrm{T} 4$. It is worthwhile to investigate why $\mathrm{T} 3+\mathrm{T} 3, \mathrm{~T} 3+\mathrm{T} 2$ and $\mathrm{T} 3+\mathrm{T} 4$ undergo tone sandhi consistently no matter whether they are leftaligned or right-aligned, but $\mathrm{T} 1+\mathrm{T} 1, \mathrm{~T} 4+\mathrm{T} 1$ and $\mathrm{T} 4+\mathrm{T} 4$ undergo sandhi inconsistently depending on their positions in trisyllables. T3+T3, T3+T2 and T3+T4 start with T3, which is the only dip-rising tone in Tianjin and is more complex than the other three lexical tones. $\mathrm{T} 1+\mathrm{T} 1, \mathrm{~T} 4+\mathrm{T} 1$ and $\mathrm{T} 4+\mathrm{T} 4$ start with $\mathrm{T} 1$ or $\mathrm{T} 4$, which is a simple falling tone and less complex than T3. We propose that the different behaviors between T3 and T1/T4 can be attributed to the difference in tonal complexity.

According to Yip (2002: 27-30), the tonal complexity order is rising, falling, high and low, as shown in (17), where $>=$ more complex than.

(17) Order of tonal complexity (Yip 2002:27-30)

Rising $>$ Falling $>$ High $>$ low

Tianjin T3 in Chao number is 214, as opposed to simple falling tones T1 (41) and T4 (53), and therefore T3 (214) is more complex than T1 (41) and T4 (53).

Zhang (2002) studies the correlation between duration and contour-bearing ability and indicates that the greater the number of pitch targets of a tone, the longer duration it requires. T3 is a dip-rising tone, which has three pitch targets. So it requires longer duration than T1 and T4, which are simple falling tones and requires only two pitch targets. Our analysis here is consistent with the correlation between duration and contour-bearing ability in Zhang (2002). T3 requires longer duration than T1 or T4, so it cannot keep its underlying tone unless it is on the prosodic head.

Our analysis in which T3 cannot keep its underlying tone unless it is on the prosodic head is also supported by the behavior of T3 in Tianjin disyllabic sequences. The fact that T3 changes when it is in front of T2, T3 and T4 in disyllables indicates that T3 tends to lose its underlying tone unless it is on the prosodic head. T3 seems to be more unstable than any other lexical tones in Tianjin because $\mathrm{T} 3$ is the only tone in Tianjin that has been reported to undergo changes when it is in front of T2, T3 and T4 in disyllables. Although T3 has not been reported to undergo categorical changes when it is in front of $\mathrm{T} 1$, it shows major interspeaker and intraspeaker variations when it is in front of T1. Our acoustic data indicate that $\mathrm{T} 3$ is reduced when it is in front of T1. It seems that T3 cannot fully keep its underlying tone when it is on the first syllable in disyllabic sequences, which is consistent with our analysis that T3 can only keep its underlying tone when it is on the prosodic head.

T3 behaves differently from T1 and T4 in disyllables not only in Tianjin, but also in Mandarin dialects. T3 sandhi $(\mathrm{T} 3+\mathrm{T} 3 \rightarrow \mathrm{T} 2+\mathrm{T} 3)$ is the prevalent tone sandhi rule in Mandarin dialects. First, T3 sandhi rule exists in most Mandarin dialects, though their pitch values are different in different dialects. Second, T3 sandhi rule is the only tone sandhi rule in some Mandarin dialects. The third reason is from a perspective of diachronic phonetics. T3 sandhi 
rule has existed in Northern Mandarin since $16^{\text {th }}$ century (Mei, 1977), and therefore it is a common tone sandhi rule development seen from a diachronic perspective.

We suggest that as the most complex tone in Tianjin, T3 keeps its full tonal contour only when it occupies the prosodic head, i.e. the third syllable with the primary stress. Therefore, T3 in either the first or second syllable in a trisyllabic sequence consistently undergo tone sandhi in a left-to-right application. The derivations in (18) illustrate the differences in behavior between $\mathrm{T} 3$ and $\mathrm{T} 1$.

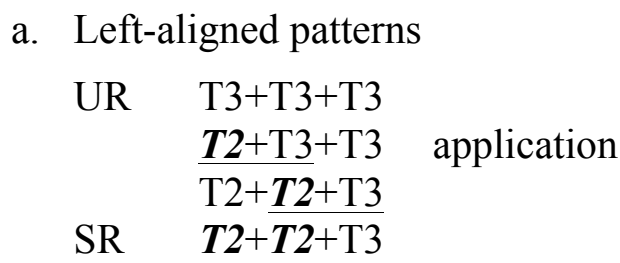

b. Left-aligned patterns

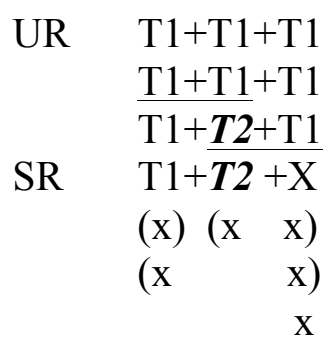

In $\mathrm{T} 3+\mathrm{T} 3+\mathrm{T} 3$, neither the first $\mathrm{T} 3$ nor the second $\mathrm{T} 3$ is on the prosodic head, and therefore they both undergo tone sandhi when their sandhi conditions are satisfied, as shown in (18a). $\mathrm{T} 1$ is less complex than $\mathrm{T} 3$ and it can keep its underlying tone unless it is on a weak syllable, so the first $\mathrm{T} 1$ does not undergo tone sandhi in $\mathrm{T} 1+\mathrm{T} 1+\mathrm{T} 1$, as shown in (18b). This difference in tonal complexity can account for why $\mathrm{T} 3$ always undergoes tone sandhi no matter whether the relevant sequence is left-aligned or right-aligned, but whether T4 and T1 undergo tone sandhi is sensitive to their metrical position in a trisyllabic sequence.

4. Conclusions. Our phonological analysis concludes that both the conflicting directionality and underapplication issues in Tianjin trisyllabic tone sandhi disappear if metrical structures and differences in tonal complexity are taken into consideration. We propose that tone sandhi applies consistently from left to right in trisyllables. Metrical conditions and tonal complexity can account for the inconsistent application of $\mathrm{T} 1+\mathrm{T} 1, \mathrm{~T} 4+\mathrm{T} 1$ and $\mathrm{T} 4+\mathrm{T} 4$ and the consistent application of $\mathrm{T} 3+\mathrm{T} 3, \mathrm{~T} 3+\mathrm{T} 2$ and $\mathrm{T} 3+\mathrm{T} 4$ in trisyllables, and therefore resolve conflicting directionality and underapplication issues. $\mathrm{T} 1+\mathrm{T} 1, \mathrm{~T} 4+\mathrm{T} 4$ and $\mathrm{T} 4+\mathrm{T} 1$ do not undergo tone sandhi when they are left-aligned in a trisyllabic sequence because T1 and T4 do not lose their underlying tones unless they are on the weak syllable. This metrical account is further supported by a trisyllabic sequence with a final neutral tone, which renders the final syllable unstressed and leads to tone sandhi application at the left edge. The consistent applications of the $\mathrm{T} 3+\mathrm{T} 3, \mathrm{~T} 3+\mathrm{T} 2$ and $\mathrm{T} 3+\mathrm{T} 4$ rules regardless of edge alignment in a trisyllabic sequence are attributed to tonal complexity. $\mathrm{T} 3+\mathrm{T} 3, \mathrm{~T} 3+\mathrm{T} 2$ and $\mathrm{T} 3+\mathrm{T} 4$, either they are left-aligned or right-aligned, undergo sandhi in trisyllables because T3 is the most complex tone in Tianjin and it tends to lose its underlying tone when it is not on the prosodic head and when its sandhi conditions are satisfied. As the most complex tone in Tianjin, T3 routinely undergoes tone sandhi unless it occupies the prosodic head, i.e. the third syllable with the primary stress.

Tianjian trisyllabic tone sandhi has long been considered a difficult and complex system to analyze (Li \& Liu 1985, Chen 1986, Chen 2000, Lin 2008, among others). Our study not only illuminates the true nature of Tianjian tone sandhi patterns, but also provides a case study where metrical structure and tonal properties interact to influence tone sandhi rule applications beyond the typical disyllabic domain. 


\section{References}

Chan, Marjorie Kit-Man. 1985. Fuzhou phonology: a non-linear analysis of tone and stress. Seattle, WA: University of Washington dissertation.

Chen, Matthew Y. 1986. The paradox of Tianjin tone sandhi. Proceedings of the Chicago Linguistic Society Meeting (CLS) 22. 98-114.

Chen, Matthew Y. 2000. Tone sandhi: Patterns across Chinese dialects. Cambridge: Cambridge University Press.

Duanmu, San. 1995. Metrical and tonal phonology of compounds in two Chinese dialects. Language 71(2). 225-259. http://www.jstor.org/stable/416163.

Huang, Liangxi, Yan, Xiuhong \& Lu, Jilun. 2005. Tianjin fangyanli de tunyin xianxiang (The elision in the Tianjin dialect). Yuyan Kexue (Linguistic Sciences) 4. 66-75.

Hyman, Larry M. 2007. Universals of tone rules: 30 years later. In Tomas Riad \& Carlos Gussenhoven (eds.), Tones and tunes, vol.1: Typological studies in word and sentence prosody. 1-34. Berlin \& New York: Mouton de Gruyter.

Li, Qian \& Chen, Yiya. 2016. An acoustic study of contextual tonal variation in Tianjin Mandarin. Journal of Phonetics 54(2). 123-150. https://doi.org/10.1016/j.wocn.2015.10.002.

Li, Xingjian \& Liu, Sixun. 1985. Tianjinhua de liandu biandiao (Tone sandhi in the Tianjin dialect). Zhongguo Yuwen (Studies of the Chinese Language) 4. 76-80.

Lin, Hui-shan. 2008. Variable directional applications in Tianjin tone sandhi. Journal of East Asian Linguistics 17(3). 181-226.

Lu, Jilun. 1997. Tianjin fangyan zhong de yizhong xin de liandu biandiao (A new tone sandhi rule in Tianjin dialect). Tianjin Shida Xuebao (Journal of Tianjin Normal University) 4. $67-72$.

Mei, Tsu-lin. 1977. Tones and Tone Sandhi in $16^{\text {th }}$ Century Mandarin. Journal of Chinese Linguistics 5(2). 237-260.

Milliken, Stuart, Zhang, Guangping, Zhang, Xueyi, Li Zhiqiu \& Lu, Ying. 1997. Resolving the paradox of Tianjin tone sandhi. In Jialing Wang \& Norval Smith (eds.), Studies in Chinese phonology. 53-80. Berlin \& New York: Mouton de Gruyter.

Shi, Feng. 1986. Tianjin fangyan shuangzizu shengdiao fenxi (Analysis of disyllabic tones in Tianjin dialect). Yuyan Yanjiu (Linguistic Research) 6 (1). 80-93.

Shi, Feng \& Wang, Ping. 2004. Tianjinhua shengdiao de xin bianhua (New changes in Tianjin tones). In Feng Shi and Zhongwei Shen (eds.), The joy of research: a festschrift in honor of professor William S.-Y. Wang on his seventieth birthday. 176-188. Tianjin: Nankai University Press.

Wang, Jialing. 2002. Youxuanlun he Tianjinhua liandu biandiao ji qingsheng (OT and Tianjin tone sandhi and neutral tone). Zhongguo Yuwen (Studies of the Chinese Language) 4. 363-371.

Wang, Jing \& Wang, Lijia. 1993. Putonghua duo yinjie ci yinjie shi chang fenbu moshi (The types of relative lengths of syllables in polysyllabic words in Putonghua). Zhongguo Yuwen (Studies of the Chinese Language) 2. 112-116.

Wang, Xiaomei. 2003. Tianjin fangyan sanzizu de liandu biandiao (Tianjin trisyllabic tone sandhi). Zhongguo Yuwen (Studies of the Chinese Language) 2. 165-171.

Wang, Xiaomei. 2015. A sociolinguistic study of Tianjin tone sandhi. East Lansing, MI: Michigan State University manuscript.

Wang, Xiaomei \& Lin, Yen-Hwei. 2016. Metrical structure and tone sandhi: evidence from Ei tonal reduction. TAL 2016: Proceedings of the fifth international symposium on tonal aspects of languages. 70-74. 
Wang, Xiaomei \& Lin, Yen-Hwei. 2017. No more conflicting directionality: Metrical conditions on Tianjin Chinese trisyllabic tone sandhi. Proceedings of the 2016 annual meeting on phonology. Linguistic Society of America. https://doi.org/10.3765/amp.v4i0.3993.

Wang, Zhije Jenny. 1999. Cihui biandiao, cifa biandiao he yinxi biandiao (Lexical tone change, morphological tone change and phonological tone change). In Xu Liejiong (eds.), Gongxi yu gexing (Universality and specificity). 288-310. Beijing: Beijing Language and Culture University Press.

Wee, Lian-Hee. 2004. Inter-tier correspondence theory. New Brunswick, NJ: Rutgers University dissertation.

Wee, Lian-Hee. 2015. Prominence from complexity: Capturing Tianjin ditonal patterns. Language and Linguistics 16(6). 891-926. 10.1177/1606822X15602614

Wright, Martha Susan. 1983. A metrical approach to tone sandhi in Chinese dialects. Amherst, MA: University of Massachusetts dissertation.

Yan, Jingzhu \& Lin, Maocan. 1988. Beijinghua sanzizu zhongyin de shengxue biaoxian (The acoustic correlates of the stress in Beijing trisyllabic sequences). Fangyan (Dialects) 3. 227-237.

Yip, Moira. 2002. Tone. Cambridge \& New York: Cambridge University Press.

Zhang, Jie. 2002. The effects of duration and sonority on contour tone distribution. New York: Routledge.

Zhang, Jie \& Liu, Jiang. 2011. Tone sandhi and tonal coarticulation in Tianjin Chinese. Phonetica 68(3). 161-191. https://doi.org/10.1159/000333387. 